\title{
Laminiertes Zinndioxid - Restrukturierte FSP Schichten für Sensoranwendungen
}

\author{
Jens A. Kemmler ${ }^{1}$, Sven Schopf ${ }^{2}$, Lutz Mädler ${ }^{2}$, Nicolae Barsan ${ }^{1}$, Udo Weimar ${ }^{1}$ \\ ${ }^{1}$ Institut für Theoretische und Physikalische Chemie, Universität Tübingen, Auf der Morgenstelle 15, \\ D-72076 Tübingen,
}

$\underline{\text { Jens.Kemmler@ipc.uni-tuebingen.de }}$

${ }^{2}$ Stiftung Institut für Werkstofftechnik, Universität Bremen, Badgasteiner Str. 3, D-28359 Bremen

\begin{abstract}
Kurzfassung:
Die Sensormaterialherstellung kann eine sehr langwierige Angelegenheit darstellen. Um die bei der Herstellung einhergehenden Probleme zu umgehen und Sensormaterialien möglichst schnell her zu stellen wird hier die Flammensprühpyrolyse genutzt. Mittels dieser Synthesemethode erhält man sehr schnell hochporöse Schichten von Metalloxiden. Diese Schichten weißen Porositäten von bis zu 98\% auf und sind mechanisch sehr instabil. Hier zeigen wir die Auswirkungen der Restrukturierung der Oberfläche durch Laminierung in Bezug auf den Grundwiderstand des Sensors und die sensorischen Eigenschaften der Schichten in Abhängigkeit vom gewählten Laminierdruck und von Oberflächendotierung durch $0.1 \%$-wt Palladium.
\end{abstract}

Schlüsselwörter: Zinndioxid, Chemoresistor, Laminierte Schichten, Transferverfahren, Sensorherstellung

Der konventionelle Weg zur Sensorherstellung, für Sensoren basierend auf halbleitenden Metalloxiden ist ein material- und zeitintensives Unterfangen. Ausgehend von einem gewöhnlichen, auf Zinndioxid $\left(\mathrm{SnO}_{2}\right)$ basierenden Gassensor müssen einige Stufen abgearbeitet werden, um das gewünschte Material in die Hände zu bekommen. Die herkömmlichen Wege führen in aller Regel über Sol-Gel-Chemie, gefolgt von zeitaufwändigen Kalzinierungsprozessen um ein geeignetes Material zu erhalten. Abhängig von den gewünschten Eigenschaften des Materials kann dies Wochen in Anspruch nehmen.

Eine neuere Methode Metalloxide in einem einzelnen Syntheseschritt und damit praktisch ohne Zeitaufwand zu erzeugen stellt die Flammensprühpyrolyse (FSP) dar.[1,2] Durch die Pyrolyse wird ein metallorganischer Vorläufer in das korrespondierende Metalloxid überführt. Die Textur dieses Metalloxides definiert sich hauptsächlich durch nanodispergierte Einkristalle, welche thermophoretisch auf einem gewünschten Substrat abgeschieden werden können.[2,3] Diese so erzielten chemosensitiven Schichten zeichnen sich durch hohe Porositäten von nahezu $98 \%$ aus. Die dadurch entstehende effektive Oberfläche ist enorm (bis zu 160 $\mathrm{m}^{2} / \mathrm{g}$ )[3], führt aber auch zu sehr niedrigen elektrischen Leitwerten, it est hohen Widerständen. Die hier vorgestellte Arbeit befasst sich mit den sensorischen Eigenschaften von undotiertem und mit Palladium dotiertem Zinndioxid, welches durch FSP hergestellt und umgehend durch Laminierung auf konventionelle Sensorsubstraten überführt wurde.

Die Idee des Laminierens dieser Materialien ist sehr viel neuer. Hintergrund der Untersuchung ist der Versuch die hochporösen Schichten zu stabilisieren, da das direkt abgeschiedene Material äußerst fragil gegen Mechanische 
Beschädigungen ist (Partikel, physischer Kontakt,...).

Der hier angewendete Laminierprozess basiert auf einem Sheet-to-Sheet Prozess, durchgeführt bei Raumtemperatur und niedrigen Laminierdrücken. Das durch FSP auf einem Filter abgeschiedene Material wird mittels Druck auf ein Substrat übertragen und dabei komprimiert. Wie Schopf et. al. bereits zeigen konnten, kann so eine mechanisch restrukturierte und damit stabilisierte Schicht erzeugt werden. Die Messung der Porengrößenverteilung hat dabei gezeigt, dass es zu einer Homogenisierung der Schichten kommt [4].

\section{Experimentelles}

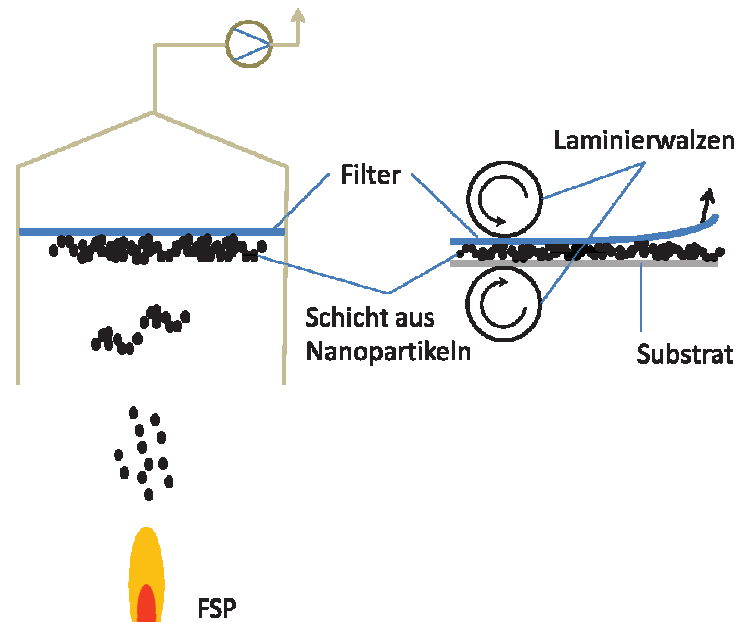

Abb. 1. Ablauf der Beschichtung und der anschließenden Übertragung der Nanokristalle auf ein Sensorsubstrat. Im ersten Schritt kommt es zur Bildung und Ansammlung des Materials, im zweiten wird das Material auf ein Sensorsubstrat laminiert und der Filter entfernt.

Der Prozess der Flammensprühpyrolyse ist gut dokumentiert und muss hier nicht näher wiederholt werden $[1,2,3]$.

Die sensitive Schicht wurde durch ein konventionelles Rollenlaminiergerät vom Filter zum Substrat übertragen.

Die hier vorgestellten Messwerte wurden unter der Nutzung einer dynamischen Gasmischanlage erzielt. Als Sensorantwort wird eine Widerstandsänderung der sensitiven Schicht erwartet, dementsprechend wurde der Widerstand mittels eines Digitalmultimeters (Keithley 199) aufgezeichnet. Um die Oberflächenkinetik zu beschleunigen und das Material in einen halbleitenden Zustand zu bekommen wurde die Sensoren mittels rückseitig angebrachter Heizer auf $300^{\circ} \mathrm{C}$ Betriebstemperatur gehalten. Mittels der Gasmischanlage wurden stabile
Testatmosphären eingestellt, zum einen in trockener Atmosphäre (>50 ppm), zum anderen unter feuchten Bedingungen (50 \% rel. Feuchte; $\left.20{ }^{\circ} \mathrm{C}\right)$. Der Gasfluß wurde stets konstant bei 200 sccm gehalten.

Die Veränderungen der Morphologie wurden rein optisch und mithilfe einer FIB/SEM (FEI DB235) Untersuchung durchgeführt. Um Veränderungen so gut wie möglich auszuschließen wurde versucht die Schichten ohne Goldbesputterung durchzuführen. Die Beschleunigungsspannung für die lonensäule (Magnum) betrug $30 \mathrm{kV}$, der Strahlstrom wurde entsprechend an den Querschnitt angepasst (20 nA Grobschnitt; 3nA Feinschnitt). Für die Bildaufnahme wurde die Beschleunigungsspannung entsprechend einem Kompromiss aus Auflösung und der Verhinderung von Aufladungseffekten gewählt.

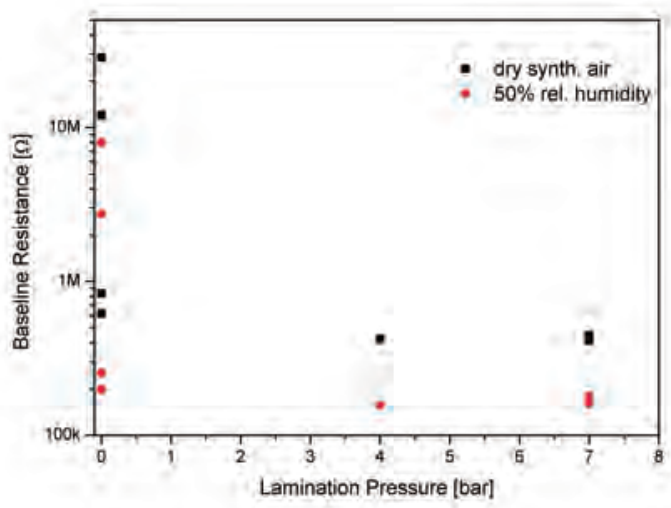

Abb. 2. Grundwiderstand in Abhängigkeit des Laminierdruckes in trockener (schwarze Quadrate) und feuchter (rote Punkte) Atmosphäre. Das direkt Abgeschiedene Material zeigt einen deutlich höheren Grundwiderstand, wohingegen eine Komprimierung der Schicht zu einer Senkung desselben führt.

\section{Vorunteruchungen über das Verhalten von laminierten Schichten}

Für Voruntersuchungen wurde die Laminierung von Nanopartikeln auf eine handelsübliche Aluminiumfolie durchgeführt. Durch die ebene Oberfläche der Folie wurden so Unebenheiten wie beispielsweise durch die Interdigitalelektroden auf den Sensorsubstraten vorhanden, umgangen. Diese Voruntersuchungen sollen zeigen, inwiefern der Druck die Schichtdicken beeinflusst. Die Ergebnisse zeigen, dass eine Erhöhung des Laminierdrucks einhergeht mit einer Verringerung der Schichtdicke (Tab.1, Abb.3). 


\begin{tabular}{|c|c|c|c|c|}
\hline & $\begin{array}{c}\text { Probe 2 } \\
{[\mu \mathrm{m}]}\end{array}$ & $\begin{array}{c}\text { Probe 3 } \\
{[\mu \mathrm{m}]}\end{array}$ & $\begin{array}{c}\text { Probe 5 } \\
{[\mu \mathrm{m}]}\end{array}$ & $\begin{array}{c}\text { Probe 6 } \\
{[\mu \mathrm{m}]}\end{array}$ \\
\hline $\begin{array}{c}\text { Messung } \\
\# 1\end{array}$ & 36,45 & 36,84 & 26,33 & 27,77 \\
\hline $\begin{array}{c}\text { Messung } \\
\# 2\end{array}$ & 29,42 & 32,5 & 24,59 & 27,1 \\
\hline $\begin{array}{c}\text { Messung } \\
\# 3\end{array}$ & 44,85 & 36,07 & 27,29 & 34,33 \\
\hline $\begin{array}{c}\text { Messung } \\
\# 4\end{array}$ & 40,03 & 33,85 & 24,21 & 29,8 \\
\hline $\begin{array}{c}\text { Messung } \\
\# 5\end{array}$ & 46,39 & 29,13 & 29,22 & 23,64 \\
\hline $\begin{array}{c}\text { Messung } \\
\# 6\end{array}$ & 45,23 & 35,2 & 32,98 & 30,67 \\
\hline Mittelwert & 40,40 & 33,93 & 27,44 & $\mathbf{2 8 , 8 9}$ \\
\hline SDV (x) & 5,99 & 2,57 & 2,99 & 3,31 \\
\hline Median & 42,44 & 34,53 & 26,81 & 28,79 \\
\hline
\end{tabular}

Tab. 1: Auf jeder Probe wurde an 6 separaten Stellen die Schichtdicke untersucht. Die Laminierdrücke für die Proben sind entsprechend: 1(Probe 2), 2.5, 4, 5.5 und 7(Probe 6) Bar.

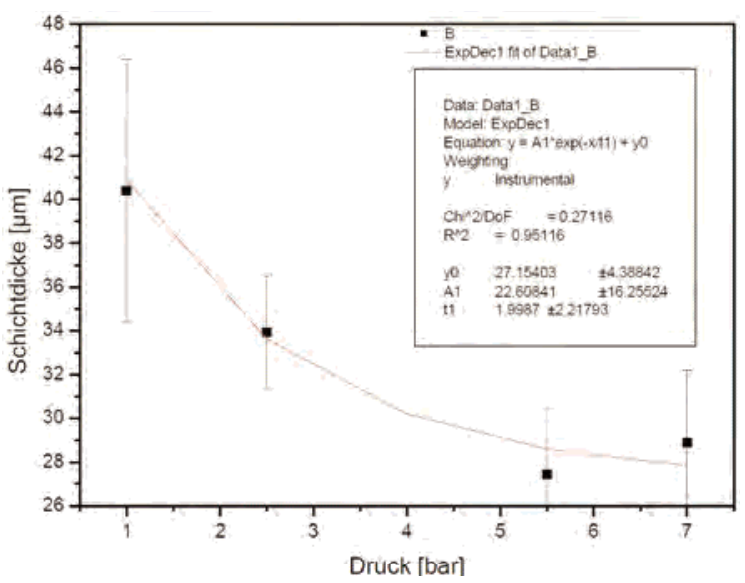

Abb. 3. Schichtdicken verhalten in Abhängigkeit des gewählten Laminierdrucks.

\section{Schichtdicke in Abhängigkeit vom Laminierdruck}

Durch die Komprimierung der sensitiven Schicht kommt es nun zur Ausbildung wesentlich kürzerer, elektrisch leitender Pfade und somit zu einer Absenkung des Grundwiderstandes der sensitiven Schicht. Wie in Abbildung 2 gezeigt konnte diese Vermutung auch entsprechend nachgewiesen werden.

Die hohe Porosität der sensitiven Schicht ist gewünscht, führt jedoch bei der Untersuchung der Morphologie mithilfe von FIB/SEM zu Problemen. Durch die hohe Porosität der Schichten kommt es bei der Querschnittsherstellung zur Ausbildung sog. FIB-Artefakte, sichtbar als säulenartige Strukturen (Abb.3, rechts).

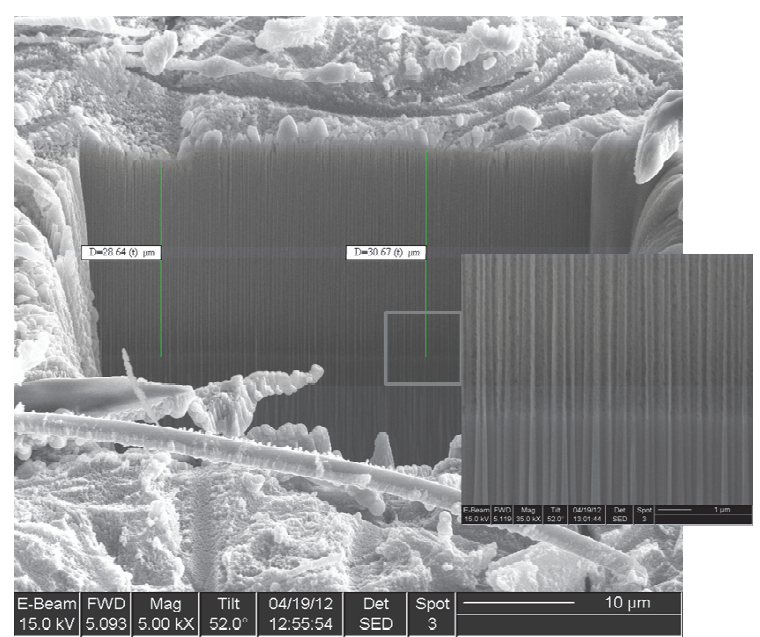

Abb.4: FIB-Querschnitt in eine mit 7bar laminerte Schicht. Am unteren Bildrand lässt sich klar eine mitlaminierte Glasfaser erkennen. Am rechten Bildrand aufgezeigt ist ein Beispiel für die bei porösen Strukturen typischerweise auftretenden säulenartigen Artefakte.

Auch bei den laminierten Sensoren zeigt sich, dass der anliegende Druck sich entsprechend der Vorversuche auf die Schichtdicke des Sensormaterials auswirkt. Auffällig ist weiterhin, dass, wie auch bei den Vorversuchen, teilweise Glasfasern aus dem Abscheidungsfilter mit in die sensitive Schicht übertragen werden. Dies kann man beispielsweise an Abbildung 4 sehen.

Auch beim Übergang, dem laminieren auf das Sensorsubstrat treten ähnliche Probleme auf, wie in Abbildung 4 zu sehen werden auch hier Teile des Glasfaserfilters übertragen.

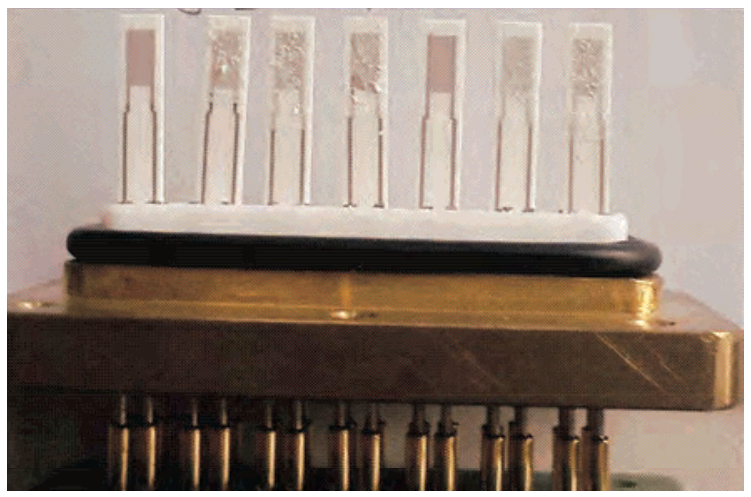

Abb.4: Die Abbildung gibt einen optischen Eindruck der Oberfläche der laminierten Sensoren wieder. VInr: Direkt abgeschiedenes Material (dd), 0,4,7 bar, dd, 4,7 bar.

\section{Sensorische Eigenschaften}

Um die Leistungsfähigkeit der Schichten hinblicklich ihrer sensorischen Eigenschaften zu charakterisieren wurde das Verhalten im Bezug auf ein oxidierendes (Stickstoffdioxid, $\mathrm{NO}_{2}$ ) und 
ein reduzierendes (Kohlenmonoxid, CO) Testgas studiert. In Abbildung 5 aufgeführt die entsprechenden Ergebnisse.

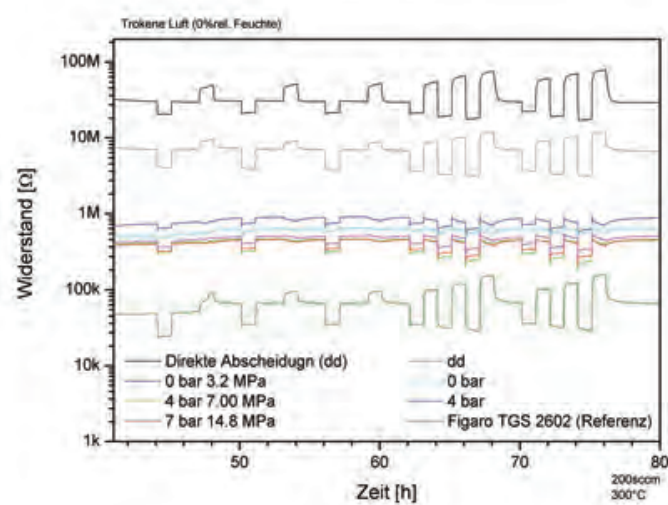

Abb.5: Verlauf des Widerstandes über die Zeit. Die Sensoren wurden alternierend Konzentrationen von100 ppm CO und 1 ppm $\mathrm{NO}_{2}$ ausgesetzt. Im späteren Verlauf der Messung wurde die Konzentration von CO auf 300 ppm erhöht und die von $\mathrm{NO}_{2}$ auf $2.4 \mathrm{ppm}$.

Wie zu erkennen ist, zeigen die laminierten Sensoren ein schlechteres Ansprechverhalten gegenüber $\mathrm{CO}$ als die direkt abgeschiedenen Sensoren. Gleichwohl zeigt das undoierte Material im Trend, dass eine erhöhter Laminierdruck zu einer besseren Leistung führt.

Setzt man die Sensoren einer oxidierenden Atmosphäre wie $\mathrm{NO}_{2}$ aus, so zeigt sich, dass die direkt abgeschiedenen Sensoren sich normal verhalten. $\mathrm{SnO}_{2}$ als n-typ Halbleiter verzeichnet in einer oxidierenden Atmosphäre eine positive Änderung des Widerstandes, er steigt. Interessanter Weise reagieren die laminierten Sensoren auf diese Änderung fast überhaupt nicht. Auch mit dem Abgleich der Literatur ist ein solches Veralten für einen n-Typ Halbleiter nicht zu erwarten.

\section{Verbesserung des Laminierprozesses}

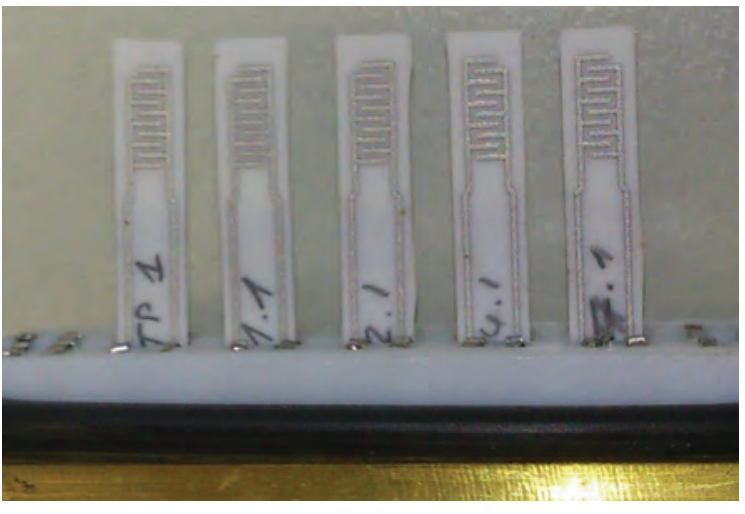

Abb. 6: Visuelle Darlegung der Oberflächenbeschaffenheit der Sensoren. VInr: Direkt abgeschiedenes Material (dd), 1,2,4,7 bar.
Um das Problem der verbleibenden Glasfasern zu überwinden wurde in der Folge eine Abscheidung der Partikel auf Metallfilter bevorzugt. Der Prozess an sich wurde beibehalten. Als zusätzliche Änderungen wurde das Material mit 0.1-wt\% Pd dotiert, sowie Sensorsubstrate mit niedrigerem Aspekt Verhältnis gewählt. Durch die Dotierung soll eine größere Oberflächenaktivität erreicht werden und eine damit einhergehende höhere Sensitivität auf CO.

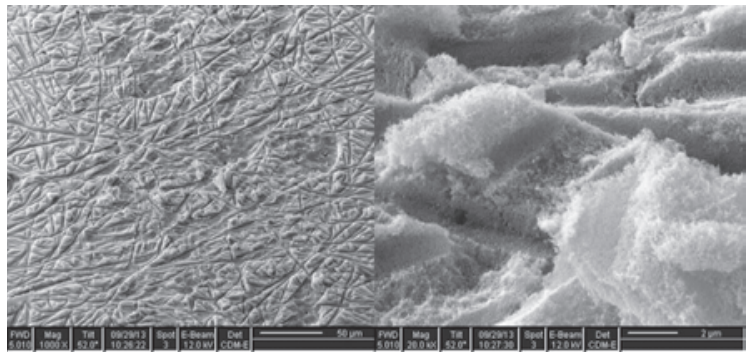

Abb. 7: FIB-Querschnitt in eine mit 4 bar laminerte Schicht. Es sind keine mitlaminierten Fasern erkennbar, lediglich die Abdrücke der Filterfasern.

Eine optische Untersuchung der Proben zeigt keinerlei Störungen der Oberfläche durch mitlaminierte Strukturen. Die Oberfläche ist lediglich durch die Oberflächenstruktur des Filters beeinflusst, da dessen Topographie durch die Laminierung als Negativ eingeprägt wird (vgl. Abb. 7).

Aufgrund des kleineren Aspekt Verhältnisses der Elektrodenstruktur, steigt die Anzahl der Back-to-Back Schottky Grenzen und damit der Grundwiderstand der sensitiven Schicht.

Allgemein lässt sich festhalten, dass das neue Material auf sämtliche Stimuli stärker reagiert als das undotierte Material. Im Bezug auf die Reaktion auf ein reduzierendes Gas, wie hier CO, steigt das Sensorsignal $\left(R_{0} / R\right)$ von ca. 1,4 auf 5. Auch die Sensitivität gegenüber $\mathrm{NO}_{2}$ konnte durch die Dotierung wieder hergestellt werden. Es läßt sich festhalten, dass die Sensitivität des Sensors abhängig vom Laminierdruck steigt. Mit einer Erhöhung des Laminierdrucks bis 4 bar, oder $2.5 \mathrm{MPa}$ effektivem Druck, steigt die Sensitivität, ehe sie bei höheren Drücken wieder abfällt, vgl. Abbildung 6 . 


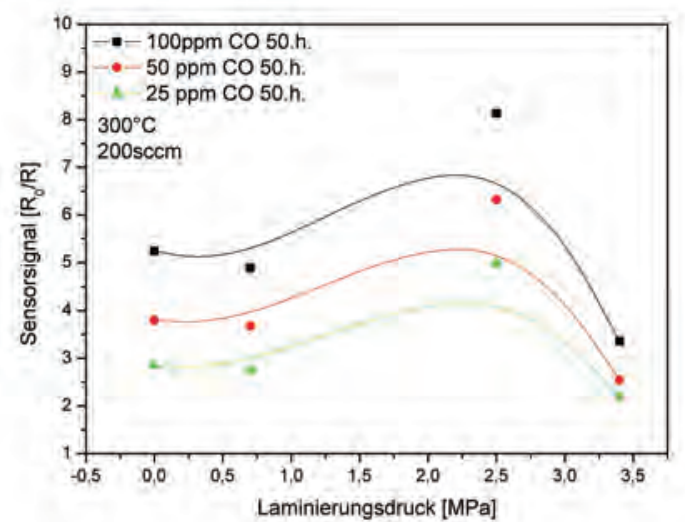

Abb. 6: Gezeigt ist die Abhängigkeit des Sensorsignals vom effektiven Laminierdruck. Der Sensor ist in diesem Fall betrieben bei $300^{\circ} \mathrm{C}$ und 200 sccm Gasfluß.

Im Fall eines oxidierenden Testgases wie $\mathrm{NO}_{2}$ verhält sich das Material etwas anders. Hier zeigt sich keinerlei Abhängigkeit der Sensitivität vom Laminierdruck, über die gesamte getestete Breite verhalten sich die Sensoren sehr ähnlich. Der größte Effekt resultiert in diesem Fall aus der Betriebstempereratur. So wird die größte Sensorantwort stets bei niedrigen Temperaturen erhalten.

\section{Schlussfolgerungen}

Die hier gezeigten Materialien besitzen klar unterschiedlich Eigenschaften. Während das pure Zinndioxid unter Kompression eine Selektivität entwickelt zeigt sich das dotierte Material recht unbeeinflusst. Im Falle des dotierten Materials scheint eine Kompression der Strukturen einen positive Effekt zu haben und das Material scheint für $\mathrm{CO}$ zugänglicher zu werden. Nicht zu vergessen, auf der anderen Seite steigt durch die höhere Kompression die effektive Temperatur der sensitiven Schicht, so dass auch eine Reaktion mit $\mathrm{CO}$ bevorzugt ablaufen wird. Diese Erhöhung basiert auf der höheren Wärmeleitfähigkeit der komprimierten Masse.

Im Falle des Nichtansprechens auf $\mathrm{NO}_{2}$ lässt sich bisweilen kein Unterschied im Austauschverhalten von Oberflächenaktiven Hydroxispezies feststellen (DRIFTS). Anhand der FIB/SEM Aufnahmen lässt sich aber auch nicht zeigen, dass ein Filtereffekt erzielt werden kann - Selektivität durch Filtration - die Strukturen sind immernoch hoch porös, so dass Stickstoffdioxid ohne Probleme sämtliche Stellen in der Schicht erreichen kann. Dies zeigen auch die Messungen mit dotiertem Material. Hier konnte $\mathrm{NO}_{2}$ zweifelsfrei nachgewiesen werden, unabhängig vom gewählten Laminierdruck. Diese Ergebnisse führen zu dem Schluss, dass allein die Oberflächenaktivität eine Änderung erfahren haben muss. Leider konnten wir bis dato den Grund hierfür spektroskopisch noch nicht nachweisen.

\section{Quellenverzeichnis}

[1] L. Mädler, H.K. Kammler, R. Mueller, S.E. Pratsinis, Controlled synthesis of nanostructured particles by flame spray pyrolysis, J. Aerosol Sci. 33 369-389 (2002).

http://dx.doi.org/10.1016/S0021-8502(01)00159-8

[2] T. Sahm, L. Mädler, A. Gurlo, N. Barsan, S.E. Pratsinis, U. Weimar, Flame spray synthesis of tin dioxide nanoparticles for gas sensing, Sens. Actuator B-Chem. 98 (2-3) 148-153 (2004). http://dx.doi.org/10.1016/j.snb.2003.10.003

[3] J. A. Kemmler, S. Pokhrel, J. Birkenstock, M. Schowalter, A. Rosenauer, N. Bârsan, U. Weimar, L. Mädler, Quenched, nanocrystalline $\mathrm{In}_{4} \mathrm{Sn}_{3} \mathrm{O}_{12}$ high temperature phase for gas sensing applications, Sens. Actuators B: Chem. 161 (1) 740-747 (2012) http://dx.doi.org/10.1016/j.snb.2011.11.026

[4] S.O.Schopf, S. Salameh, L. Mädler, Transfer of highly porous nanoparticle layers to various substrates through mechanical compression, Nanoscale 5 (9), 3764-3772 (2013). dx.doi.org/10.1039/C3NR34235B 